\title{
Development of a reliable estimation procedure of radioactivity inventory in a BWR plant due to neutron irradiation for decommissioning
}

\author{
Ken-ichi Tanaka, ${ }^{1,2 *}$ and Jun Ueno ${ }^{3}$ \\ ${ }^{1}$ The Institute of Applied Energy, 105-0003 Tokyo Japan \\ ${ }^{2}$ Tokyo Institute of Technology, 152-8550 Tokyo Japan \\ ${ }^{3}$ Genden Information System Company, 105-0004 Tokyo Japan
}

\begin{abstract}
Reliable information of radioactivity inventory resulted from the radiological characterization is important in order to plan decommissioning planning and is also crucial in order to promote decommissioning in effectiveness and in safe. The information is referred to by planning of decommissioning strategy and by an application to regulator. Reliable information of radioactivity inventory can be used to optimize the decommissioning processes. In order to perform the radiological characterization reliably, we improved a procedure of an evaluation of neutron-activated materials for a Boiling Water Reactor (BWR). Neutron-activated materials are calculated with calculation codes and their validity should be verified with measurements. The evaluation of neutron-activated materials can be divided into two processes. One is a distribution calculation of neutron-flux. Another is an activation calculation of materials. The distribution calculation of neutron-flux is performed with neutron transport calculation codes with appropriate cross section library to simulate neutron transport phenomena well. Using the distribution of neutron-flux, we perform distribution calculations of radioactivity concentration. We also estimate a time dependent distribution of radioactivity classification and a radioactive-waste classification. The information obtained from the evaluation is utilized by other tasks in the preparatory tasks to make the decommissioning plan and the activity safe and rational.
\end{abstract}

\section{Introduction}

Four commercial nuclear power reactors are undergoing decommissioning in Japan. Adding to the four reactors, five operating organizations declared to shut down permanently their six nuclear power reactors and to transit to decommissioning-stage in last fiscal year. Furthermore some reactors plan to start decommissioning in the next decade.

In Japan, a regulatory body requires an application whenever any organization of nuclear facility starts decommissioning. We call tasks for the application preparatory tasks for decommissioning. The preparatory tasks consist of five tasks as seen in Fig. 1; these are a radiological characterization, an investigation for plant characteristics, a planning for decommissioning activity, a safety assessment for decommissioning and a cost estimation for decommissioning. First two tasks, which are the radiological characterization and the investigation for plant characteristics, are called a plant characterization. These two should be performed in the earliest stage of the preparatory tasks. Outputs from them are referred to by other tasks. A reliability of an evaluation of radioactivity inventory, which is performed in the radiological characterization, is much important to plan optimized decommissioning planning and also to accomplish decommissioning activities safely and rationally [1].

The radiological characterization consists of three sub-tasks; these are an evaluation for neutron-activated materials, an evaluation for contaminated materials and an amount investigation of waste materials. Outputs from them are stored in database so as to be utilized by other tasks easily.

In order to perform the radiological characterization reliably, we improved a procedure of the evaluation of neutron-activated materials. The evaluation is composed of two calculation processes. One is a distribution calculation of neutron-flux [2, 3]. Another is a distribution calculation of radioactivity [4].

\footnotetext{
* Corresponding author: k-tanaka@iae.or.jp
} 
To accomplish the calculations reliable, we need to verify them. Distributions of neutron-flux and radioactivity are calculated by using calculation codes and verified with measurements.

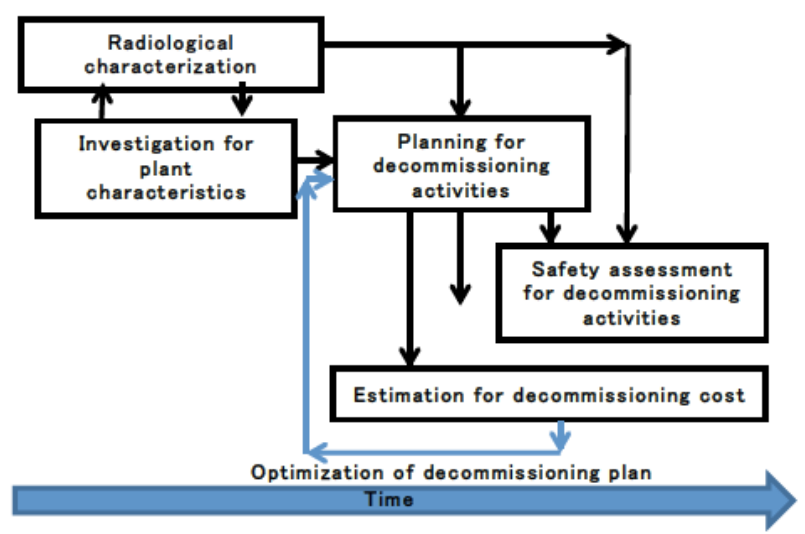

Fig. 1. Flow diagram of preparatory tasks

\section{The procedure of the evaluation for neutron-activated materials.}

\subsection{Outline of the procedure}

In order to perform the radiological characterization reliably, we improved a procedure of the evaluation for neutron-activated materials for a BWR. We perform four works in the procedure of the evaluation of neutronactivated materials as shown in Fig. 2. These are measurement of neutron-flux by using activation foils, distribution calculations of neutron-flux in a Primary Containment Vessel (PCV) by using calculation codes, distribution calculations of neutron-flux outside the PCV and time dependent distribution of radioactivity.

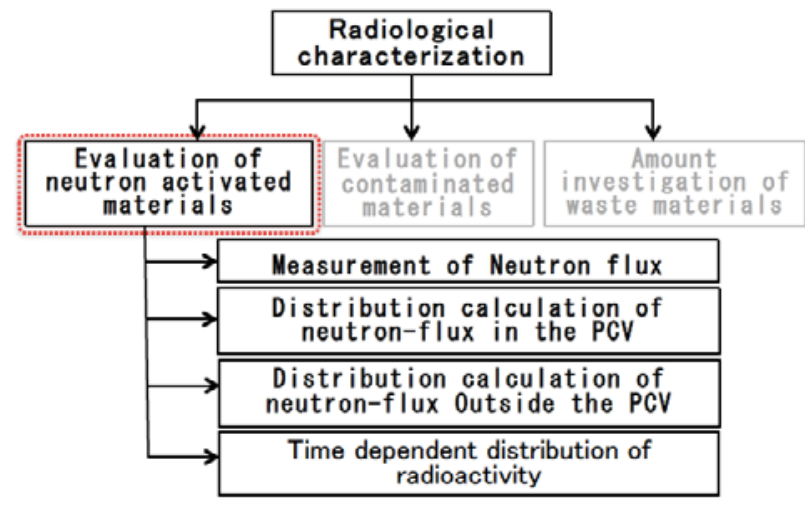

Fig. 2. Sub-tasks in Radiological characterization and activities in evaluation for neutron-activated material

Calculations are verified with measurements. By considering comparisons between calculations and measurements (C/Ms), we modified the calculation model repeatedly until our goal of reliability is achieved. When $\mathrm{C} / \mathrm{Ms}$ are within one to ten, we judge that calculations are reliable and our goal of reliability is achieved [5].

\subsection{Measurements of radioactivity due to neutron irradiation}

We measured radioactivity in a range where neutron irradiation should be considered. The range is in the PCV and in a Main Steam line tunnel room (MS tunnel room). We performed measurements with activation foils. We implemented the measurements for the following two reasons. One was to obtain knowledge about both distribution of radioactivity and distribution of neutron-flux. Another was to verify validity of calculations.

We set activation foils at 30 locations where the characteristics of distribution of neutron-flux such as streaming, leakage, shielding, diffusion and others could be observed in the PCV as shown in Fig. 3. As seen in the right panel of the figure, six locations, these were from No.19 to No.24, were set to observe an effect of neutron streaming through a Feed Water (FW) piping hatch.

We used three kinds of foils as neutron activation detectors; these were Gold foil (Au), Gold foil cover by Cadmium $(\mathrm{Au}+\mathrm{Cd})$ and Nickel foil $(\mathrm{Ni}) . \mathrm{Au}$ and $\mathrm{Au}+\mathrm{Cd}$ were used to measure neutron-flux in thermal energy range and in epi-thermal energy range [6]. We also use $\mathrm{Ni}$ foil that is threshold detector to measure neutron-flux in fast energy range [6].

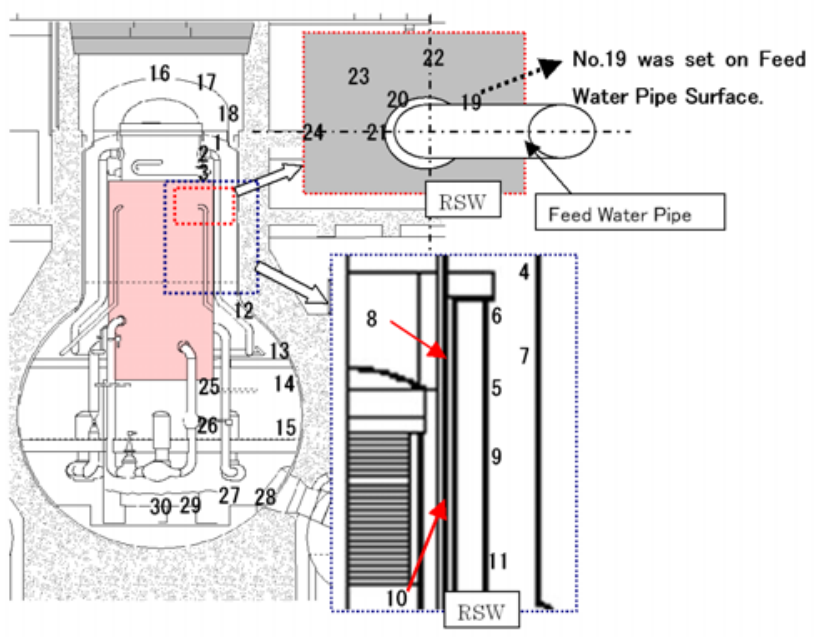

Fig. 3. Measurement locations of activation foils in the PCV

In order to measure neutron-fluxes that streams through the Biological Shielding Wall (BSW) both along a Main Steam line (MS line) and a Feed Water line (FW line) and that diffuses into the MS tunnel room, We also set ten foils in the MS tunnel room as seen in Fig. 4. In a simulation of neutron transport phenomena, a reliability of a downstream side in the phenomena depends on that of an upstream. In case of this measurement, the reliability of the estimation in the MS tunnel room would prove that in the BSW.

We set six sets of activation foils, which were No.1, 2, 3, 5,6 and 10, around the MS line to observe neutron streaming through the MS line penetrating the BSW. One set, No.4, was located near the sidewall of the room. Other three sets, No.7, 8, and 9, were located on an aisle 
in an entrance of the MS tunnel room. These four foil sets were set to measure diffusion of neutron-flux in the MS tunnel room.

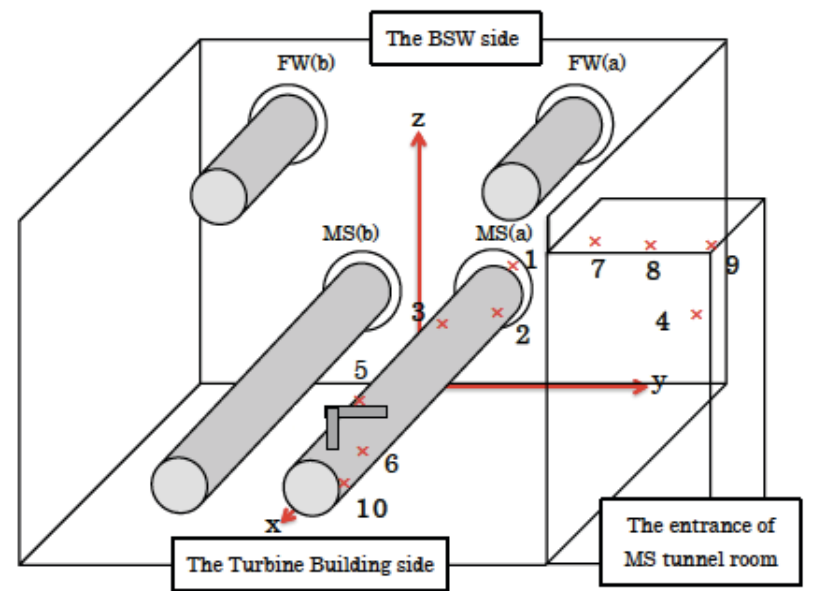

Fig. 4. Measurement locations of activation foils in the MS tunnel room

\subsection{Distribution calculations of neutron-flux}

We calculated three-dimensional (3D) distribution and two-dimensional (2D) distribution of neutron-flux around a reactor of the BWR in the PCV and inside the BSW. Furthermore, we performed 3D distribution calculation around the MS line and the FW line that penetrate the BSW and in the MS tunnel room. Areas where we set the ranges for these calculations are shown in Fig. 5.

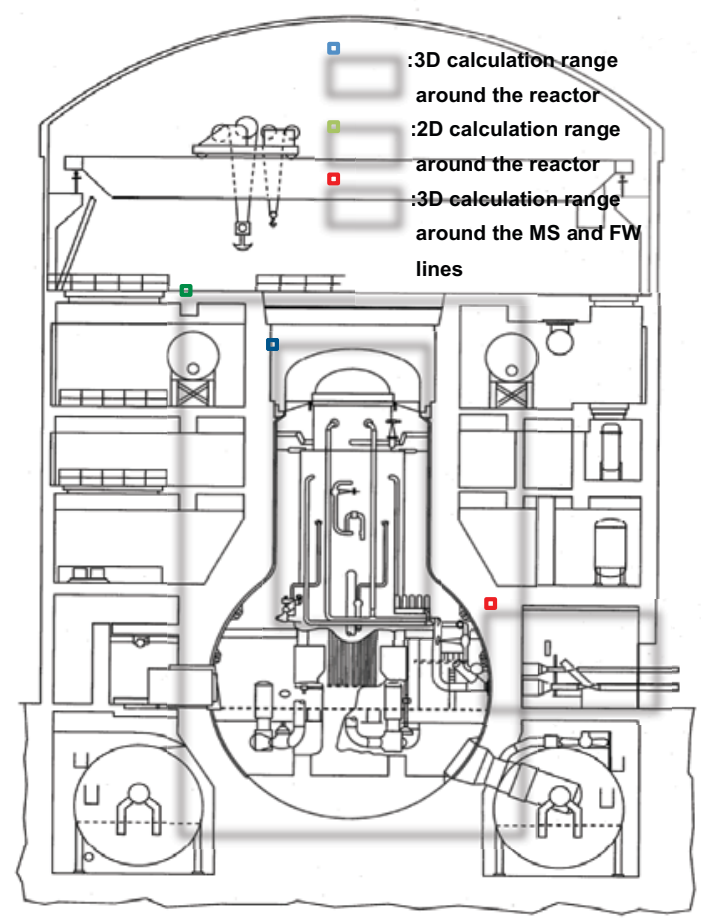

Fig. 5. Calculation ranges of $3 \mathrm{D}$ and $2 \mathrm{D}$ calculations

\subsection{1 $3 D$ distribution calculation of neutron-flux around the reactor in the PCV}

We performed 3D distribution calculation of neutronflux around the reactor in the PCV by using 3D Discrete
Ordinate method (Sn) calculation code TORT [2, 7]. A range of the calculation was an area surrounded with a blue square in Fig. 5. A purpose of the 3D calculation was to obtain knowledge of a characteristic of distribution of neutron flux. We set the range of the 3D calculation where important phenomena of neutron transport can be observed. We modeled the FW piping penetrating a Radiological Shielding Wall (RSW) that makes heterogeneity in neutron-flux distribution and so on. Both assessing the measurements and observing the 3D distribution of neutron-flux provided knowledge of the characteristics of distribution of neutron-flux in the PCV.

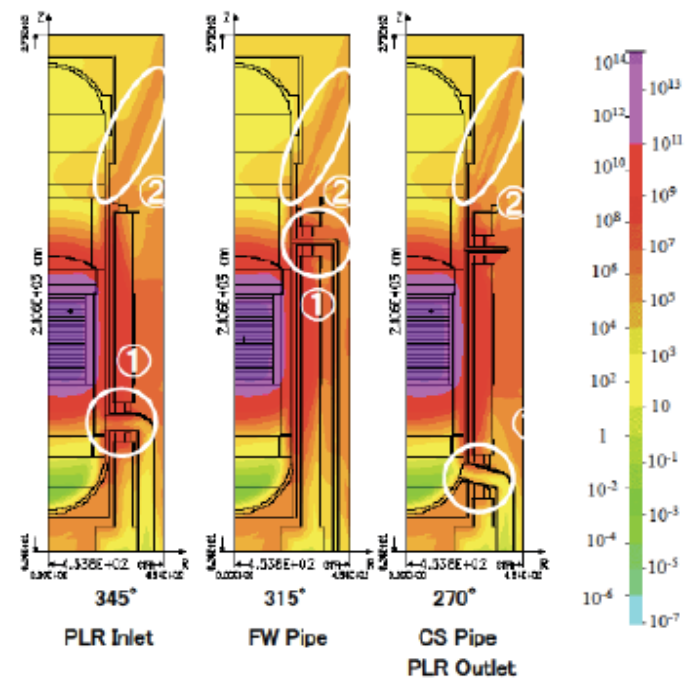

Vertical $(\mathrm{R}-\mathrm{Z})$ Plane
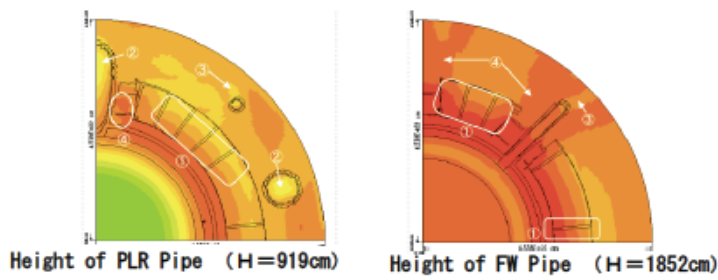

Hor izontal Plane

Fig. 6. Calculated 3D distribution of neutron-flux on vertical planes and on horizontal planes

We can find the following characteristics of neutronflux distribution by observing contour maps of neutronflux on r-z plane (the upper panel) in Fig. 6.

1) Although neutron leaks from hatches, water in the piping reduces amount of neutron leakage.

2) Although neutron streams through the gap between a Reactor Pressure Vessel (RPV) and a Radiological Shielding Wall (RSW), a stabilizer reduces amount of neutron streaming. The streaming neutron forms the area where flux level is rather high in the upper part of the PCV but the area is limited to a narrow range.

The lower panel in Fig. 6 also shows contour maps on $r-\theta$ plane. The heights of a plane in the left and the right panels are $919 \mathrm{~cm}$ and $1852 \mathrm{~cm}$ from the bottom of the PCV, respectively. Following characteristics are observed. 
1) H-Beam in the RSW forms azimuthal heterogeneity in the distribution.

2) Water in the piping forms the area where flux level is rather low in the piping.

3) Shield effect of water in the piping forms the area where rather low flux behind the piping but the area is limited in a narrow range.

4) Although neutron leaks from hatches, water in the piping reduces amount of neutron leakage.

\subsubsection{D distribution calculation of neutron-flux in the PCV}

We calculated 2D distribution of neutron-flux with 2D Sn code DORT [2, 7]. A range of the calculation was an area surrounded with a green square in Fig. 5. In order to perform the reliable $2 \mathrm{D}$ calculation of neutronflux, we needed to generate the 2D model that could simulate neutron transport phenomena well in the PCV and to set optimized calculation conditions. At the onset, we generated a simple model, and causes of difference between calculated and measured fluxes were investigated. Then, we modified the $2 \mathrm{D}$ models by utilizing the knowledge obtained from measurements and $3 \mathrm{D}$ calculation.

The 2D distribution calculation started with a simple model. Only the RPV and concrete structures were modeled in the initial model. The 2D model was modified repeatedly until reliability was achieved. In order to simulate well the distribution of neutron-flux in the PCV, following phenomena were investigated and materials that would affect the $2 \mathrm{D}$ distribution of neutron-flux were taken into consideration in the final model.

1) Streaming through the gap between the RPV and the RSW.

2) Leakage from hatches and shielding effect of piping.

3) Upward streaming through the standpipes in the RPV, where steam is flowing through.

4) Shielding effect of H-Beam in the RSW.

5) Shielding (neutron absorption) effect of Control Rod Drive (CRD).

6) Shielding effect of the PCV internals in "Flask" area.

The best combination of mesh interval width and number of directional quadrature was also surveyed in the final model. Optimized calculation condition, which is the best combination of mesh interval width and numbers of directional quadrature, is as follows: 389 meshes in radial direction, 1104 meshes in axial direction and directional quadrature S16.

Fig. 7 shows that almost all of $\mathrm{C} / \mathrm{Ms}$ of total neutron-flux at 30 locations meet the criteria of reliability that $\mathrm{C} / \mathrm{M}$ is within one to ten.

Fig. 8 shows the 2D distributions of neutron-flux. The leftmost contour map shows the distribution of total neutron-flux. Contour maps of fast, epithermal and thermal neutron-flux are shown from the right neighbor side of that each in turn. The distribution characteristics of neutron-flux in the PCV, which

were investigated and modeled, are observed in these maps: streaming and leakage are remarkable in fast and epithermal energy range (areas indicated " $a$ " in the maps). Shielding effect by the PCV internals in "Flask" area affects thermal flux distribution.

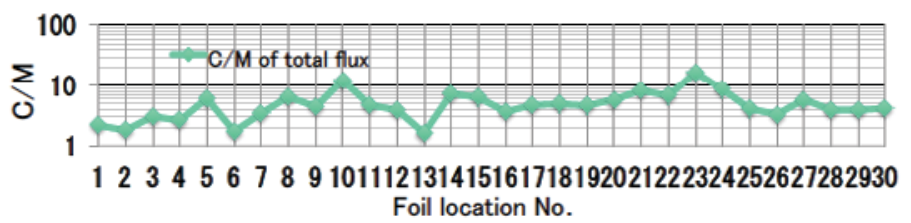

Fig. 7. Comparison between measured fluxes and 2D calculated fluxes (C/Ms)

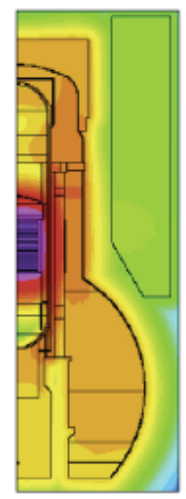

Total flux

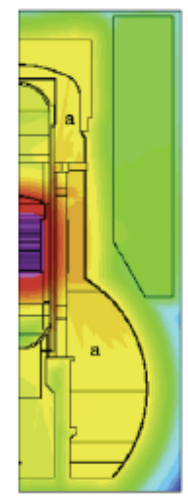

Fast flux

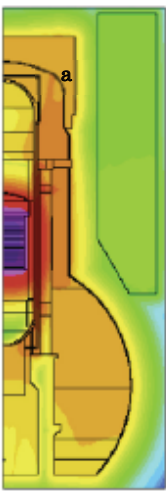

Epi-thermal flux

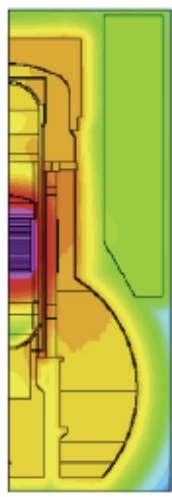

Thermal flux

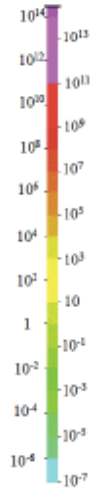

Fig. 8. Distribution maps of total flux, fast flux, epithermal flux and thermal flux

\subsubsection{D distribution calculation of neutron-flux outside the PCV}

We also calculated 3D distribution of neutron-flux outside the PCV. The area includes a region where both the MS line and FW line penetrate the BSW and the MS tunnel room. The range is surrounded with a red square in Fig. 5.

According to a report by the regulatory body in Japan, materials, which are irradiated by neutrons to an effective-dose-rate less than $6.25 \mu \mathrm{Sv} / \mathrm{hr}$, can be treated as non-radioactive materials [8]. A purpose of this 3D calculation was an estimation of a boundary that distinguishes radioactive materials and non-radioactive materials in the BSW. In our work, we call the boundary on which materials are distinguished between radioactive materials and non-radioactive (NR) materials as NR boundary.

Reliable estimation of NR boundary is crucial in preparatory tasks for decommissioning of a nuclear power plant (NPP) to determine a decommissioning scenario, a dismantling design, a disposal planning and a decommissioning cost. Especially, the reliability would influence an accuracy of estimation for amount of verylow-level concrete waste because NR boundary would be located inside concrete of a BSW of a BWR. Amount of concrete waste is important since it is much larger than that of other materials from decommissioning activity of NPP. 
The distribution calculation in the area required a separate estimation from other calculations because neutron transport phenomena in the area would be different from that of in the PCV. Neutron-streaming along the lines inside the BSW and neutron-diffusion in the MS tunnel room would occur in the area. Since these phenomena were hard to be simulated with 2D distribution calculation of neutron-flux, we performed 3D distribution calculation in the corresponding area. The calculation was performed with TORT by applying Sn-Sn coupling calculation technique $[3,7]$. We coupled the 2D calculation, which calculated neutron-flux distribution around the reactor, with the $3 \mathrm{D}$ calculation. Directional fluxes on an inner surface of the BSW given in the 2D calculation were set as Boundary Source that is one of calculation option in boundary conditions.

To simulate both neutron-streaming through the MS line and distribution of neutron-flux in the MS tunnel room well, we surveyed better combination between orders of scattering cross section and numbers of directional meshes. Because neutron-flux under epithermal energy range is dominant in the MS tunnel room, effect of order of scattering cross section (Pl) would be negligible. We judged that fine mesh set of the Levelsymmetry directional mesh set such as S16 sets or biased directional mesh set was necessary so that calculation met the criteria for reliability.

According to $\mathrm{C} / \mathrm{Ms}$ in the MS tunnel room, the calculation with S16 and P5 was the most effective to estimate NR boundary around the MS and the FW lines in our work. $\mathrm{C} / \mathrm{Ms}$ in the $\mathrm{MS}$ tunnel room at ten locations are shown in Table 1 . We judged that calculations were carried out with reliability by considering characteristics of distribution of neutron-flux in the MS tunnel room even if some $\mathrm{C} / \mathrm{Ms}$ did not meet our criteria mentioned above. Nevertheless calculations resulted that NR boundary was located in the BSW concrete and that materials in the MS tunnel room were considered to be non-radioactive as seen in Fig. 9.

Table $1 \mathrm{C} / \mathrm{Ms}$ at foil locations in the MS tunnel room

\begin{tabular}{|c|c|c|c|c|c|c|c|c|c|}
\hline Foil location & 1 & 2 & 3 & 4 & 5 & 6 & 7 & 8 & 9 \\
\hline C/M & 1.1 & 3.1 & 4.8 & 4.1 & 2.5 & 0.4 & 0.9 & 0.9 & 1.4 \\
\hline
\end{tabular}

* Radioactivity at No.10 location was not detected.

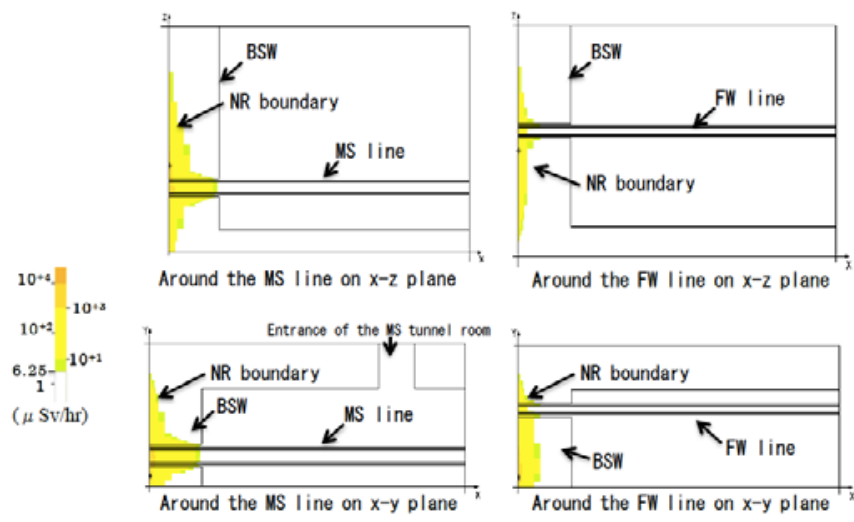

Fig. 9. NR boundary around the MS line and FW line

\subsection{Distribution calculation of radioactivity due to neutron irradiation}

We developed a new estimation procedure of time dependent distributions of radioactivity induced by neutron irradiation [9]. We performed activation calculations, which calculated generations and depletions of radioactive-nuclides due to neutron irradiation at whole spatial meshes of the 2D distribution calculation of neutron-flux. The calculations with the procedure could also provide time dependent 2D distributions of radioactivity of depleting with time after irradiation. The procedure was developed to estimate radioactivity distribution by considering characteristics of SCALE5.1/ORIGEN-S [10] calculation method and made it possible that we could estimate radioactivity distributions of various materials in much shorter computer time than the conventional method with the same level reliability.

We applied the procedure to the estimation of time dependent distributions of radioactivity inventory due to neutron irradiation around the PCV. Using the calculation results, we drew several kinds of time dependent radioactivity distribution maps. Other tasks referred to them to optimize the decommissioning strategy.

Fig. 10 shows one example of an application of the procedure. Distribution maps in the figure show time dependent distribution maps of a classification of radioactive waste for disposal and clearance from 20 years to 25 years after shut down. Area colored in green indicates ranges of very-low-level waste and areas colored in gray indicate ranges of clearance. Areas surrounded by red dotted square indicate a location of a Primary Line Recirculation Pump (PLR pump). These maps show that we will be able to treat the PLR pump as clearance when we will dismantle it 24 year after shut down.
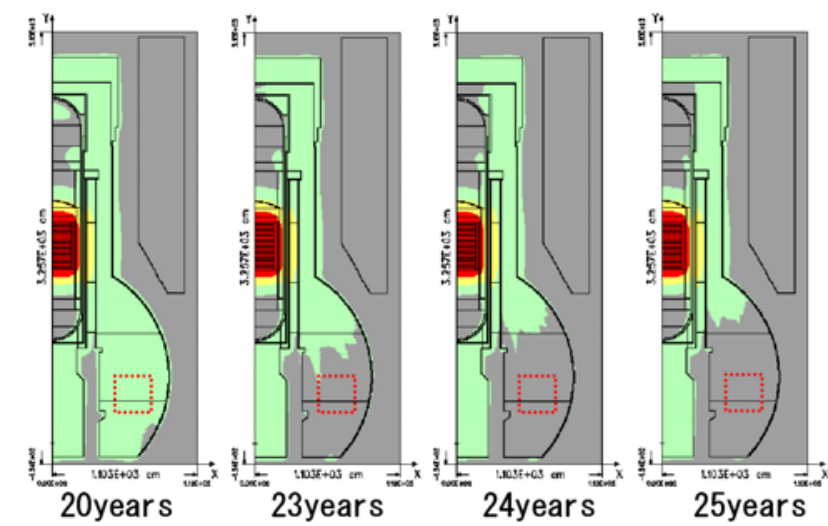

Fig. 10. Time dependent distribution maps of a classification of radioactive waste for disposal and clearance

\section{Summary}

We have developed the new procedure of evaluation for neutron-activated materials. We applied the procedure to an evaluation of radioactivity inventory of 
BWRs that were declared to transit decommissioningstage in the last fiscal year. The applications for decommissioning of BWRs have already been submitted and the regulatory body in Japan is reviewing the applications.

By applying the procedure to the BWRs, we obtained knowledge about the characteristics of distribution of neutron-flux in the PCV by measurements and by 3D distribution calculation of neutron-flux. 2D distribution calculation by referencing to the knowledge provided reliable 2D distribution of neutron-flux. The 2D distribution calculation accomplished our goal of reliability. By referencing to the $2 \mathrm{D}$ distribution of neutron-flux, we estimated time dependent distributions of radioactivity.

We also performed 3D distribution calculation of neutron-flux around the MS line and the FW line, which penetrate the BSW. We have been able to simulate neutron-streaming through the lines well. The distribution of neutron-flux around them provided that the boundary line is located in the BSW.

The information obtained from the evaluation would be utilized by other tasks in the preparatory tasks to make the decommissioning plan and the decommissioning activity safely and rationally.

The procedure of time dependent distribution of radioactivity due to neutron irradiation would play an important role in planning decommissioning strategy.

We performed this study as one of the decommissioning project promoted by JAPC. Authors thank the former director, Mr. Satoshi Karigome and the current director, Mr. Toyoaki Yamauchi for their understanding and the support for this study. We also thank members of the dismantling planning group of the decommissioning project department of JAPC for their useful information about the configuration of the internals in the PCV of Turuga NPP Unit 1 (TS-1). Members of Nuclear Service Corporation performed setting, collecting and radioactivity analysis of activation foils in the PCV of TS- 1 by the contract. We thank them for their professional works and their useful advice in the planning of the measurement locations. A computer resource was provided from Genden Information System Corporation, a subsidiary of JAPC by a contract.

\section{References}

1. METI, Roadmap for technology of LWR safety and human resource, (1997) (in Japanese)

2. www.meti.go.jp/committee/sougouenergy/

3. Tanaka K, et.al., PNE 85 (2015) 254-270, (2015).

4. Tanaka K, et.al., PNE,(to be published)

5. Tanaka K, et.al., proc. of 2013 AESJ annual meeting(2013) (in Japanese)

6. IAEA, Safety Series No.100, International Atomic Energy Agency, ISBN 9201240899, (1989).

7. Tsoulfanidis N, (Japanese translation by Sakai E), Japanese translation published by Gendaikougakusya, ISBN4874721281, (1986)

8. ORNL, CCC-650 DOORS3.2, Oak Ridge National Laboratory, (1998)

9. Advisory Committee for Natural Resources and Energy, Oct. 2007, "Report on Non-Radioactive waste from nuclear installation" (2007) (in Japanese)

10. Kurosawa M, et. al., Progress in Nuclear Science and Technology Volume 4 (2014) pp. 840 -843

11. Gauld C. I et. al., ORNL/TM-2005/39, Version 5.1, Vol. II, Book 1, Sect. F7, Oak Ridge National Laboratory, (2006) 